\title{
Pin1 overexpression is associated with poor differentiation and survival in oral squamous cell carcinoma
}

\author{
KAM-WING LEUNG ${ }^{1,4}$, CHEN-HSUN TSAI ${ }^{2}$, MICHAEL HSIAO $^{3}$, \\ CHING-JUINN TSENG ${ }^{2}$, LUO-PING GER ${ }^{2}$, KUEN-HAUR LEE ${ }^{5}$ and PEI-JUNG LU ${ }^{6}$
}

\begin{abstract}
Departments of ${ }^{1}$ Density, and ${ }^{2}$ Medical Education and Research, Kaohsiung Veterans General Hospital, Kaohsiung; ${ }^{3}$ Genomics Research Center, Academia Sinica, Taipei; ${ }^{4}$ Department of Density, Yuan's General Hospital, Kaohsiung; Institute of ${ }^{5}$ Basic Medical Sciences, and ${ }^{6}$ Clinical Medicine, National Cheng Kung University, Tainan, Taiwan
\end{abstract}

Received December 2, 2008; Accepted January 19, 2009

DOI: $10.3892 /$ or_00000329

\begin{abstract}
Phosphorylation on certain Ser/Thr-Pro motifs is a major oncogenic mechanism. The conformation and function of phosphorylated Ser/Thr-Pro motifs are further regulated by the prolyl isomerase Pin1. Pin1 has been shown to be prevalently overexpressed in human breast cancer cell lines and cancer tissues and to play a critical role in the transformation of mammary epithelial cells by activating multiple oncogenic pathways. Pin 1 expression was found to be an excellent independent prognostic marker in prostate cancer. However, little is known about Pin1 and its downstream targets B-catenin and cyclin D1 expressions in human oral cancers. In the present study, we quantified Pin1 expression in 74 paired normal/tumor human oral cancer samples as well as oral cancer cell lines. Pin1 was overexpressed in oral squamous cell carcinoma (OSCC) and its level correlated with B-catenin accumulation and cyclin D1 expression. Moreover, we examined Pin1 mRNA expression in OSCC and cancer cell lines by RT-PCR analysis. The results showed that there is concordance in the relationship between the Pin1 mRNA level and Pin1 protein expression. The up-regulation of Pin 1 mRNA expression in tumor part when comparing with that in non-tumor part was in agreement with that of the Pin1 protein overexpressed in OSCC. In addition, we showed that the molecular and immunohistochemical profiles of Pin1 overexpression were associated with progression of OSCC. Taken together, these results indicate that Pin1 is a regulator of cyclin D1 expression in OSCC and might play a role in oral oncogenesis. The overexpression of Pin1 can be used as an indicator for pathological diagnosis of OSCC.
\end{abstract}

Correspondence to: Dr Pei-Jung Lu, Institute of Clinical Medicine, National Cheng Kung University, No.1 University Rd. Tainan, Taiwan

E-mail: pjlu2190@mail.ncku.edu.tw

Key words: Pin1, oral squamous cell carcinoma, ß-catenin, cyclin D1, survival

\section{Introduction}

Pin 1 is a peptidyl-prolyl cis-trans isomerase that specifically recognizes phosphorylated Ser/The-Pro motifs and isomerizes only phosphorylated Ser/Thr-Pro (pS/T-P) peptide bonds (1-4). Previously, Pin1 has been demonstrated as the first PPIase that is essential for cell growth (3). It regulates mitosis progression and replication checkpoint in human and yeast cells $(3,5)$. Structurally and functionally, Pin 1 can be divided into N-terminus WW domain and C-terminus PPIase domain (6). The WW domain has been demonstrated to work as a phosphoserine/threonine-proline binding module that binds to phosphoproteins $(7,8)$. The PPIase domain has catalytic activity that specifically isomerizes Ser/Thr-Pro peptide bonds (4). This novel post-phosphorylation isomerase regulates the conformation of a subset of phosphoproteins such as $\mathrm{p} 53, \mathrm{Cdc} 25 \mathrm{C}$ and the microtubule-associated protein tau, thereby affecting their activity and/or protein-protein interactions $(1,9-14)$. Pin 1 has been shown to interact with a number of cancer-related phosphoproteins, which suggested Pin1 might link signal transduction to pathogenesis of cancer $(1,15,16)$. Moreover, Pin1 overexpression in several human cancers including breast and prostate and liver cancers strongly suggested that Pin 1 might play a role in tumorigenesis (16-19). Given the role of Pin1 in cell growth control and tumorigenesis, Pin 1 could represent a new anti-cancer target.

Although the molecular mechanism remains uncertain in many Pin1-overexpression related cancer types, recent studies have shown that the Pin1 protein level is correlated with tumor grade and with cyclin D1 level in human breast cancers $(16,20)$. Moreover, it has been demonstrated that Pin1 directly binds the phosphorylated Ser-Pro motif next to the APC-binding site in B-catenin, inhibits its interaction with APC and increases its translocation into the nucleus $(15,20)$. Thus, Pin 1 is a novel regulator of $\beta$-catenin signaling in breast cancer, in which APC or $\beta$-catenin mutations are not common. Cyclin D1 is a proto-oncogenic protein in the cell cycle and has been implicated in the pathogenesis of several types of cancers, including SCC $(21,22)$. Cyclin D1 gene amplification has been found in $20-50 \%$ of head and neck SCC and the protein is overexpressed in up to $80 \%$ 
cases (23-25). Conversely, cyclin D1 antisense oligonucleotides suppress SCC growth in vitro and in vivo, leading to cellular apoptosis (26). Moreover, overexpression of cyclin D1 has been found associated with a more aggressive phenotype and reduced survival in patients with head and neck SCC including tongue cancer (27-31). However, in OSCC, there were no significant differences in any of the clinical or pathological parameters and overall survival (32). These results indicate that cyclin D1 might play an important role during oncogenesis in OSCC.

The purpose of this study was to investigate whether Pin1 expression is correlated with OSCC, as well as whether Pin 1 expression is associated with prognosis of OSCC. In this report, we demonstrate that Pin 1 is overexpressed in OSCC. Our results showed that Pin1 protein overexpression is associated with the pathological stage and survival rate of OSCC. Pin 1 overexpression therefore can be used as an indicator for pathological diagnosis and prognosis in OSCC.

\section{Materials and methods}

Patients and tumor samples. Patients for immunoblotting and RT-PCR analyses: the study protocol had the approval of the ethics committee at Kaohsiung Veterans General Hospital. We examined the biopsies from 74 patients who were evaluated during 2002 and 2004 in the Department of Dentistry of Kaohsiung Veterans General Hospital, Taiwan. None had received prior chemotherapy or radiation therapy. The clinical and pathological characteristics were examined according to the standard procedure in Department of Pathology in Kaohsiung Veterans General Hospital. Histopathological diagnosis was made routinely at the Department of Pathology and tumors were classified according to the World Health Organization classification of histological differentiation.

Patients for immunohistochemistry and survival curve analyses: we reviewed the medical records of 74 patients treated for squamous cell carcinoma of the oral cavity at the Department of Dentistry of Kaohsiung Veterans General Hospital between 1998 and 2000. Clinicopathological data were obtained by retrospective chart review. Paraffinembedded tissue samples were obtained from the hospital archives. All tumors sections were confirmed being primary SCC by the Clinicopathology Department of the hospital. The mean age of the patients was 52 years (age range 27-77 years). The 74 OSCC patients were derived from the following sites: buccal mucosa $(n=34)$, tongue $(n=21)$, gingiva $(n=5)$, lip $(n=3)$, palate $(n=4)$ and others $(n=7)$. The median followup period was 54 months (follow-up range 0-86 months).

Immunoblot analysis. Four oral cancer cell lines, SAS, TW2.6 CA922, and Cal27 were obtained from Dr Mark Kuo, Department of Dentistry, National Taiwan University Hospital. Forty oral cancer tissue and its adjacent nontumor tissue specimens were used to detect Pin1 expression levels by immunoblotting. The tumor chunks were homogenized and dissolved in the sample buffer $[0.5 \mathrm{M}$ Tris- $\mathrm{HCl}$ (pH 6.8), 100\% glycerol, B-Mercaptoethanol, and $20 \% \mathrm{SDS}$ ] and boiled for $10 \mathrm{~min}$. The protein samples were electrophoresed by using SDS-PAGE and transferred onto an Immobilon ${ }^{\mathrm{TM}}-\mathrm{P}$ transfer Membranes (Millipore, Bedford, USA). The membrane was then soaked in the blocking solution (1X TBS, $0.05 \%$ Tween-20, 5\% non-fat dried milk) for overnight, incubated with anti-Pin 1 polyclonal antibody $(1: 1,000$, Oncogene Research Products, San Diego, CA, USA) diluted with 1X TBST for $2 \mathrm{~h}$ at $25^{\circ} \mathrm{C}$, and washed three times with TBST (1X TBS, $0.05 \%$ Tween-20). The membrane was then incubated with the secondary antibody (biotinylated anti-rabbit IgG, 1:5,000, Perkin-Elmer Life Sciences, Boston, MA, USA) for $1 \mathrm{~h}$ and washed with TBST three times. The enhanced chemiluminescence (ECL) system Western blotting detection reagents (Perkin-Elmer Life Science) were used according to the manu-facturer's recommendations.

Immunohistochemical analysis. Sections $(5 \mu \mathrm{m})$ were cut from the formalin-fixed and paraffin-embedded specimens. Briefly, the sections were deparaffinized in xylene and rehydrated in serial dilutions of ethanol (100, 95 and $75 \%)$ for 5 min each. Antigen retrieval was performed by autoclave treatment for $10 \mathrm{~min}$ in $0.01 \mathrm{M}$ citrate buffer ( $\mathrm{pH} \mathrm{6.4).} \mathrm{Endo-}$ genous peroxidase was quenched by treatment with $3 \%$ $\mathrm{H}_{2} \mathrm{O}_{2}$ in absolute methanol for $30 \mathrm{~min}$ at room temperature, followed by blocking of endogenous biotin activity using a biotin blocking kit (Dako Corporation, Carpinteria, CA, USA). The section were then blocked in $10 \%$ normal goat serum in Tris-buffered saline, followed by incubated overnight with Pin1 (1:100) rabbit polyclonal antibody. After $16 \mathrm{~h}$ of incubation at $4^{\circ} \mathrm{C}$, the slides were incubated for $30 \mathrm{~min}$ at room temperature with biotinylated anti-rabbit IgG antibody diluted at 1:200 with TBS containing 5\% normal goat serum. After washing twice for 5 min with PBS, the standard $\mathrm{ABC}$ process was then followed, according to the manufacturer's instructions. Diaminobenzidine was used as a chromogen, followed by counterstained with hematoxylin.

For Pin 1 protein expression levels and extent oral cancer paraffin sections were scored according to the Pin1 staining intensity in the nucleus and the extent of staining of tumor cells. Scoring was: $3(+++)$, strong staining in a normal distribution; $2(++)$, patchy or heterogeneous staining; $1(+)$, weak and fragmented; $0(+/-)$, weak or loss of staining. In addition, a score of $4(++++)$ was used to indicate intense and high staining accumulation in the nuclear/cytoplasmic fraction in tumor tissues. In normal oral epithelial cells, the staining intensity in the nucleus was weak and moderate. Therefore, high level of Pin1 staining was defined as strong (score 3) and intense (score 4) immunohistochemical staining, and low level of Pin1 as weak (score 1) and moderate (score 2) staining.

Pinl/shRNA preparation and transfection. Oral Squamous SAS and Cal27 cells were cultured in Dulbecco's modified Eagle's medium $\left(\right.$ Gibco $^{\mathrm{TM}}, \mathrm{NY}$, USA) supplemented with $10 \%$ heat-inactivated fetal calf serum incubated at $37^{\circ} \mathrm{C}$ with $5 \% \mathrm{CO}_{2}$. For experiments using cell lines, cells were transiently transfected with Pin1/shRNA (Expression Arrest ${ }^{\mathrm{TM}}$, AL, USA) using Arrest-In Transfection Reagent (Expression Arrest) in Opti-MEM for $6 \mathrm{~h}$, and then the medium was changed back to growth medium without antibiotic for 

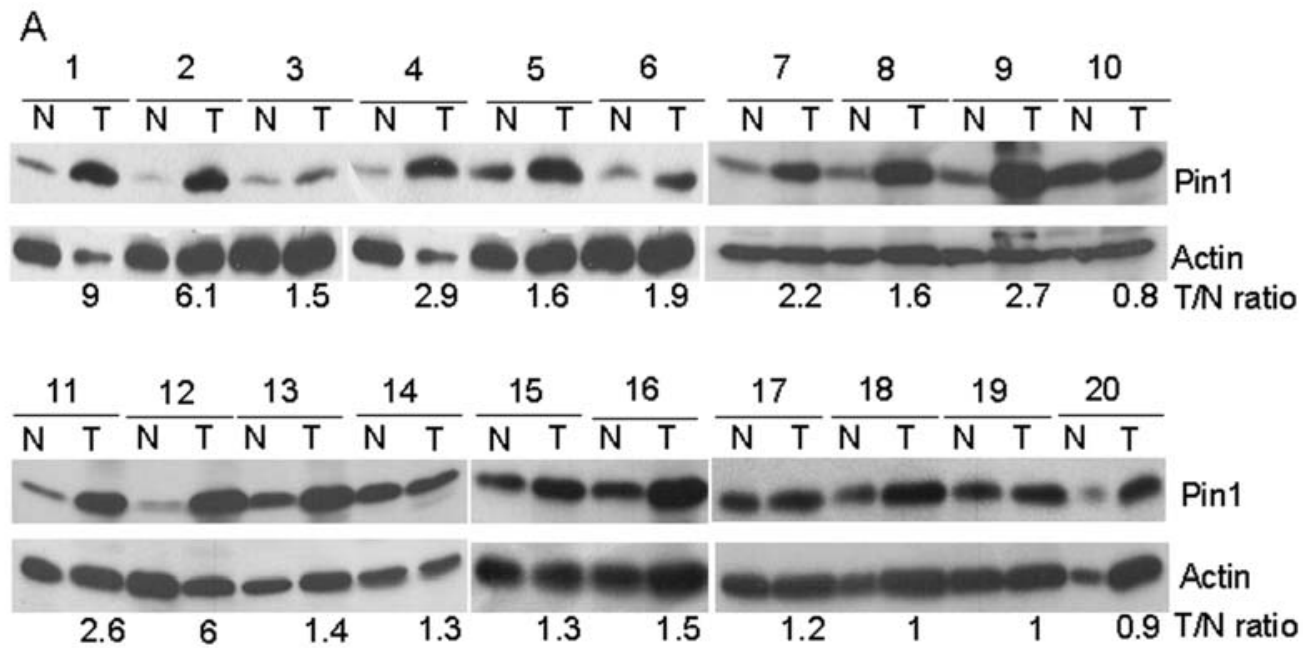

B

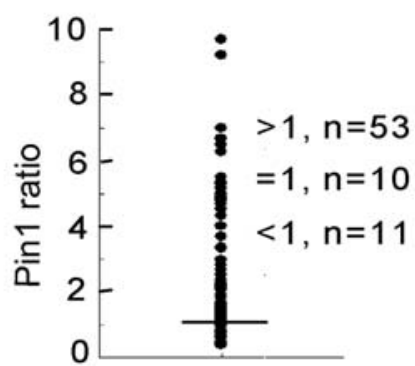

Tumor/non-tumor

C

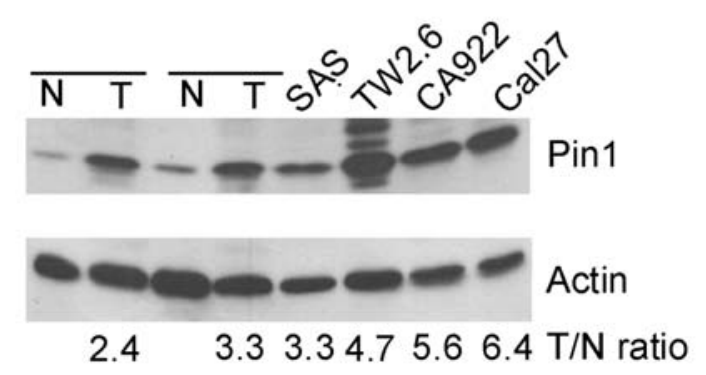

Figure 1. Examination of the Pin 1 protein expression levels in oral cancer tissues. (A) Immunoblot analysis for detecting PIN1 expression patterns in oral cancer tissue using the polyclonal rabbit anti-PIN1 and monoclonal mouse anti-actin served as an internal control. Twenty pairs of oral caner tissues (N/T pairs) were chosen and represented for total 74 pairs examined. The number underneath the tumor Pin 1 in the immunoblot membrane indicates the T/N ratio of Pin level when compared the Pin 1: actin ratio of tumor part (T) and that of its non-tumor counterpart (N). (B) Summary of the fold increase of Pin levels in OSCC. (C) Immunoblot analysis for detecting PIN1 expression patterns in four oral cancer cell lines.

additional incubation. Drug selections in SAS, Cal27 cells were performed with $3 \mu \mathrm{g} / \mathrm{ml}$ puromycin. For Western blots, time course and puromycin-selected cells were lysed in $2 \mathrm{X}$ sample buffer. Protein lysate was separated on $15 \%$ SDS-polyacrylamide gel electrophoresis and transferred to Immobilon ${ }^{\mathrm{TM}}-\mathrm{P}$ transfer Membranes (PVDF, Millipore). Membranes were blocked by 5\% skin milk in TBST buffer (10 mM Tris, pH 7.4, $150 \mathrm{mM} \mathrm{NaCl}, 0.5 \%$ Tween-20) at $4^{\circ} \mathrm{C}$ overnight, incubated for $2 \mathrm{~h}$ at room temperature with following primary antibodies: monoclonal anti- $\beta$-catenin (Santa Cruz Biotechnology, CA, USA), anti-actin (Santa Cruz Biotechnology), anti-cyclin D1 (Zymed Laboratories Inc., CA, USA), and anti-Pin1. Immunoreactive proteins were detected using Anti-Rabbit IgG, HRP-conjugated secondary antibodies (Perkin-Elmer Life Sciences) and by using the ECL system.

Statistical analysis. The relationship between Pin1, cyclin D1 and B-catenin protein expression were determined using the $\chi^{2}$ test after the expression level for each variable was dichotomized as described above. The correlation of the level of Pin 1 and the degree of cell differentiation was evaluated by spearman correlation. We used the Kaplan-Meier method to estimate survival rates and the curves. The difference between the survival curves was examined using the logrank test. Overall survival was calculated from the time of surgery to the date of death, or the date of last follow-up. All statistical analysis were performed using SPSS software (SPSS Inc., Chicago, IL, USA).

\section{Results}

Pin1 is overexpressed in primary oral cancers. To examine the role of Pin1 in OSCC, we analyzed the expression profile of Pin1 protein using 74 pairs of human non-tumor oral tissues (N) and their tumor counterparts (T) by immunoblotting analysis. Non-tumor or oral squamous cell carcinoma tissues were pulverized freshly in liquid nitrogen and the lysates were directly subjected to immunoblotting analysis with antiPin1 antibodies, followed by semi-quantification of protein levels using NIH image, as described $(12,33)$. By using antiPin1 antibodies and immunoblotting, all normal human oral tissues and their tumor parts showed immuno-reactivity. The results of 20 representative pairs of total 74 pairs of primary oral tissues examined are shown in Fig. 1A. Pin 1 was detected as an $18 \mathrm{kDa}$ protein in immunoblots, especially in tumor tissues where Pin1 was overexpressed. To compare the levels of Pin1 in each N/T pair, actin was used as an internal control and Pin1 protein expression level was expressed as Pin1: actin ratio in each examined sample. The Pin1 overexpression was defined as $\geq 50 \%$ increase when compared the Pin 1: actin ratio of tumor part with that of its non- 
A
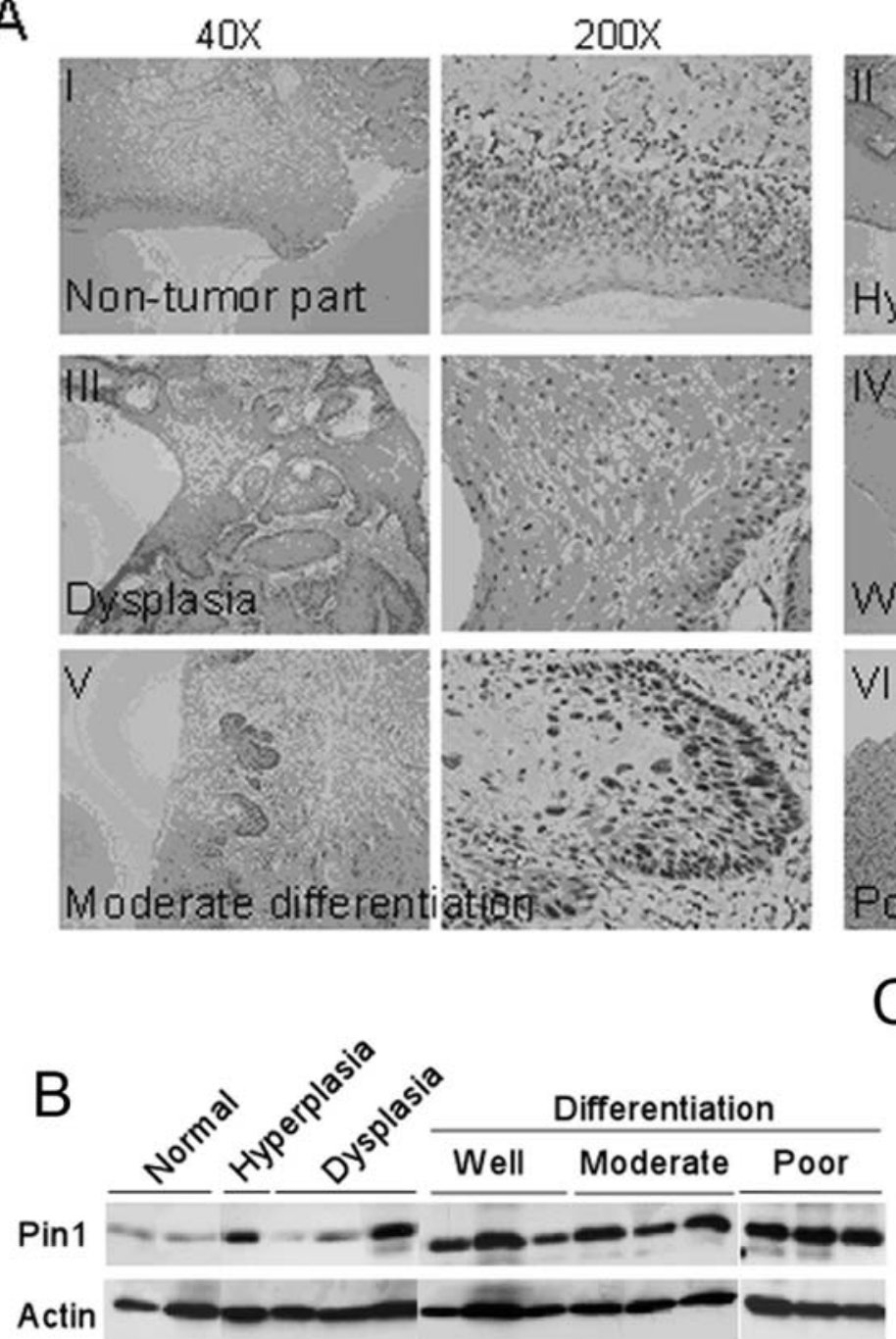
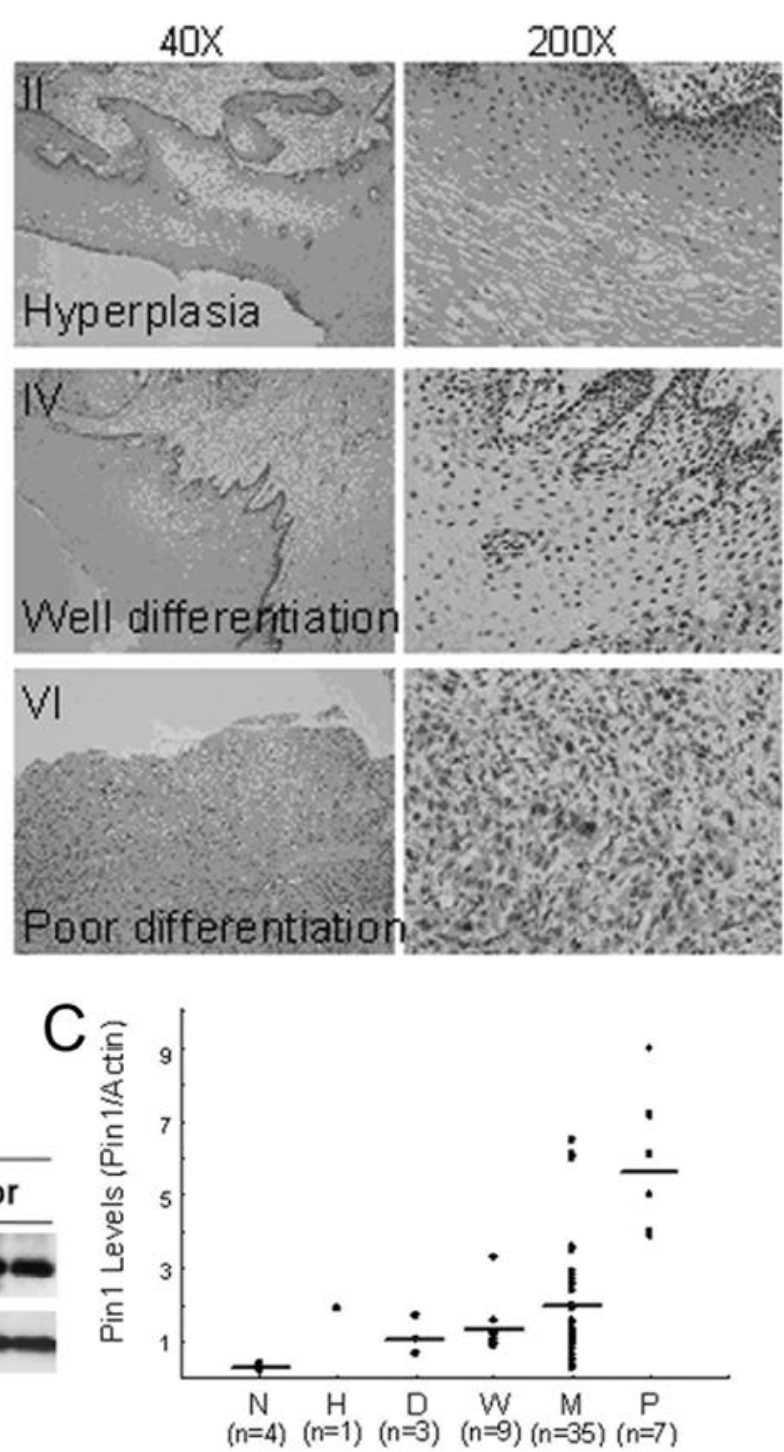

Figure 2. Pin1 protein expression level correlates with the cell differentiation status in oral squamous cell carcinoma (A-C). A (I-VI) Immunohistochemical staining patterns of Pin 1 expression in oral cancer tissues. I, normal oral mucosal epithelium treated with anti-Pin 1 antibody show weak, but detectable staining; II, hyperplasia; III, dysplasia; IV, well differentiation shows strong Pin1 staining exclusively in the nucleus; V, moderate differentiation shows high level of Pin1 staining in both nucleus and cytoplasm; VI, poor differentiation shows very intense Pin1 staining in both nucleus and cytoplasm. (B) Immunoblotting analysis of Pin1 expression in oral cancer tissues classified by cell differentiation. Antibodies specifically against Pin1 and actin were used for detecting the Pin1 and actin protein level, respectively. (C) Pin1 level analysis using the Pin1: actin ratios obtained form immunoblotting analyses.

tumor counterpart. In the total 74 pairs of oral tissues examined, 53 pairs (72\%) of oral tissues showed Pin 1 overexpression in the tumor part. The increase of Pin1 levels in 53 oral cancer pairs ranged from 0.5 - to 8.0-fold (Fig. 1B). Whereas, 21 out of 74 (28\%) oral cancer pairs did not show significant Pin1 level elevation $(n=10,13 \%)$ or even a decrease of Pin 1 protein expression $(n=11,15 \%)$ (Fig. 1A, the right bottom panel; Fig. 1B). In order to confirm the higher Pin1 level in oral cancer cells, four oral cancer cell lines derived from human oral squamous cell carcinoma were used for Pin 1 immunoblotting examination. Our results showed striking elevation from 2.3- to 5.4-fold increase of the Pin 1 protein levels in these four OSCC cell lines when compared with that in non-tumor parts (Fig. 1C). Taken together, these results indicated that Pin1 is overexpressed in human oral cancer tissue and cell lines.
Relationship between expression pattern of Pin1 and poor differentiation in OSCC patients. To further verify the Pin1 overexpression in oral tumor cells but not in normal adjacent cells, immunohistochemistry analysis was performed to examine the Pin1 distribution and expression levels in 74 human oral cancer tissues. Normal oral squamous cells showed weak but detectable Pin1 stained primarily in the nucleus (Fig. 2AI). In contrast, strong positive Pin1 nuclear stains were found in tumor cells (Fig. 2A), while weak Pin1 stains were found in surrounding normal connective tissue. It is worth mentioning that the majority of cases with medium and poor differentiation exhibited both nuclear and cytoplasmic accumulation of Pin 1 protein (Fig. 2AV and VI). In their respective non-tumor counterparts, absent to very weak cytoplasmic expression of Pin1 was observed. Interestingly, the Pin 1 level from low to high was correlated 

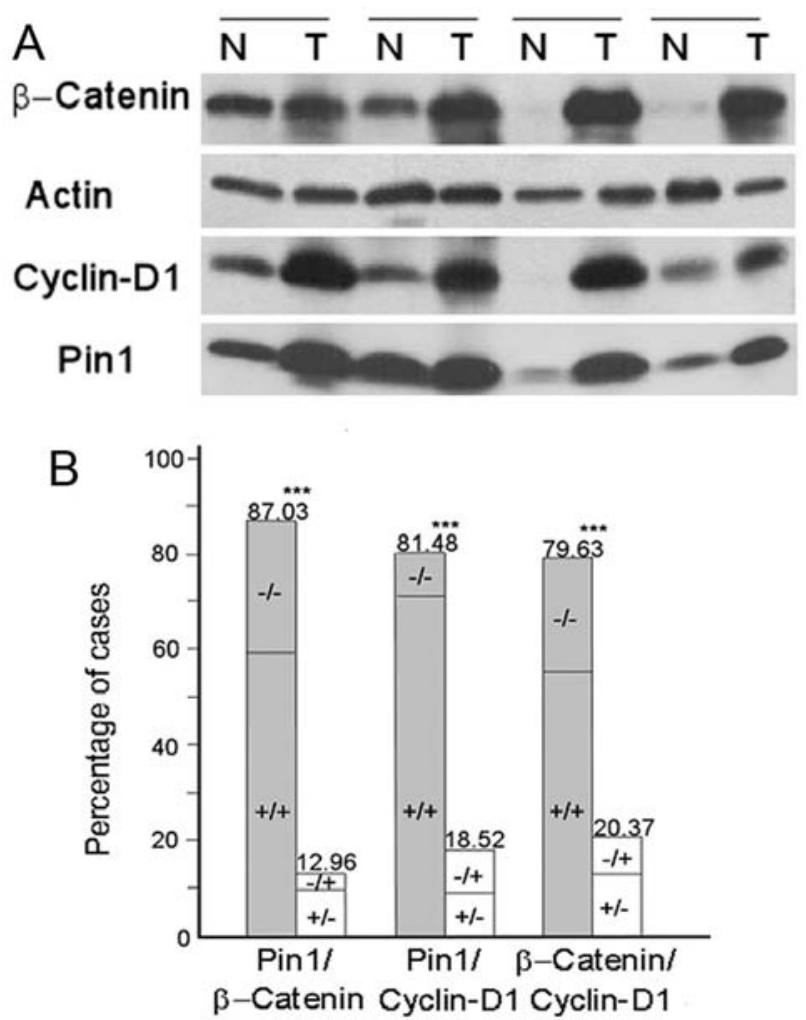

Figure 3. Expression of Pin1, ß-catenin and cyclin D1 in oral cancer tissues. (A) Immunoblot analysis for detecting Pin 1, ß-catenin and cyclin D1 expression patterns in Pin 1 overexpressing oral cancer tissue using the following antibodies- polyclonal rabbit anti-Pin 1 , monoclonal mouse anti- $\beta$ catenin or monoclonal mouse anti-cyclin D1. Monoclonal mouse anti-actin served as an internal control. (B) Concordance analysis between protein expression of Pin $1, \beta$-catenin and cyclin D1. The percentage of cases is indicated on the $y$-axis, whereas the type of comparison is shown on the $\mathrm{X}$-axis. + indicates positive protein expression (Pin1, ß-catenin and cyclin D1). Numbers above the bars indicate percentages of the total concordant group (gray sections) and non-concordant group (white sections).

with the order of pathological classification in cancer tissues from well, moderate to poor differentiation (Fig. 2AIV-VI). Moreover, we found that the Pin1 level elevation can even be detected in hyperplasia and dysplasia stages (Fig. 2AIII).

In order to confirm these results, immunoblotting was performed to examine the Pin1 protein level in oral cancer tissues. Non-tumor tissues were used as references and Pin1 level was expressed as Pin1: actin ratio as described above (16). Fig. 2B shows Pin1 overexpressed in hyperplasia, dysplasia, and cancer tissues with well, moderate, and poor differentiation status when compared with Pin1 expression level of normal oral tissue. The mean value of Pin 1 protein levels (shown in Fig. 2C), for dysplasia, well differentiation, moderate differentiation, and poor differentiation, were 1.4, 1.8, 2.2 and 5.4, respectively. Statistical analysis showed that the degree of cell differentiation was correlated with the level of Pin1 (correlation coefficient $=0.252, \mathrm{P}<0.05$ ). The conclusion drawn from the results of immunoblotting was consistent with immunohistochemistry investigation that the Pin1 protein levels correlated with the pathological poor differentiation in OSCC. These results also indicated that Pin1 level may be used as a pathological marker for oral cancer diagnosis.
Molecular signaling of Pinl overexpression related tumorigenesis in OSCC. Previously, Pin1 overexpression in breast cancers was found to stabilize $\beta$-catenin proteins and consequentially caused elevation of cyclin D1 $(16,20)$. Therefore, the expression profile of B-catenin and cyclin D1 expression were analyzed to assess their correlation with Pin1 in OSCC. Protein levels of $\beta$-catenin and cyclin D1 in 54 OSCC N/T paired specimens containing 39 pairs of Pin1-overexpressed and 15 pairs of Pin1 level down-regulated or without change were determined by immunoblotting to establish the relationship between Pin1, cyclin D1 and B-catenin protein expression in OSCC. Actin level was used as the internal control to normalize the protein amount for loading. Fig. 3A shows that Pin1, cyclin D1 and B-catenin protein levels were elevated in oral cancer tissues when compared with those in their normal counterparts. Specifically, $\beta$-catenin protein accumulation in tumor part was detected in $35 \mathrm{~N} / \mathrm{T}$ pairs accounted for $65 \%$ of total 54 pairs of OSCC specimens examined; cyclin D1 levels increased in $37 \mathrm{~N} / \mathrm{T}$ pairs accounted for $69 \%$ of total 54 pairs of OSCC specimens examined. The data of Pin1, cyclin D1 and B-catenin protein levels were then cross examined to investigate the correlation between these three parameters in OSCC. The results of statistical analysis (Fig. 3B) showed that Pin1 protein level were significantly associated with $B$-catenin level (concordance group $87.0 \%$; $\mathrm{P}<0.001$, by $\chi^{2}$ test) or cyclin $\mathrm{D} 1$ protein level (concordance group $81.5 \% ; \mathrm{P}<0.001$, by $\chi^{2}$ test), respectively. The results in Fig. $3 \mathrm{~B}$ also indicate a statistically significant correlation between $\beta$-catenin level and Cyclin D1 protein level (concordance group 79.6\%; $\mathrm{P}<0.001$, by $\chi^{2}$ test). Furthermore, our results showed 32 of $37(86 \%)$ cases with Pin1-overexpression had concomitant accumulation of $\beta$-catenin protein; 32 of 37 (86\%) had concomitant accumulation of cyclin D1 protein; and 30 of $37(81 \%)$ had both $\beta$-catenin protein and cyclin D1 protein overexpression.

In addition, the protein levels of B-catenin and cyclin D1 in four oral cancer cell lines were also examined by immunoblotting and the results are shown in Fig. 4A. All oral cancer cell lines showed the accumulation of B-catenin and up-regulation of cyclin D1 accompanying with Pin 1 overexpression when compared with normal oral tissue (Fig. 4A). Altogether, these data suggested that Pin 1 overexpression might directly lead to an increase in B-catenin and cyclin D1 levels in OSCC.

To directly demonstrate that this molecular mechanism also plays a role in OSCC tumorigenesis, a Pin 1 cDNA expression construct, Pin1-pCMV, was transiently transfected into four human oral squamous cell lines, SAS, TW2.6, CA922 and Cal27. The results from immunoblot analysis confirmed a significant increase in B-catenin and cyclin D1 levels when Pin 1 overexpressed in three cell lines SAS, TW2.6 and Cal27 (Fig. 4A). The levels of Pin 1 in the transiently transfected clones positively correlated with both ß-catenin and cyclin D1 levels. In addition to Pin1-pCMV, OSCC cells transfected with Pin 1 in various plasmids including pEGFP, pcDNA, pDsRed, also showed similar results (data not shown). These results suggested Pin1 upregulation could cause $\beta$-catenin accumulation and cyclin D1 level elevation in OSCC. This conclusion was further 


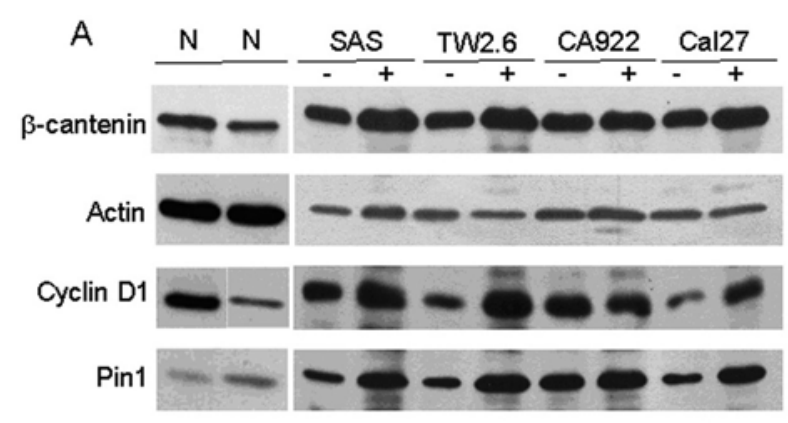

B

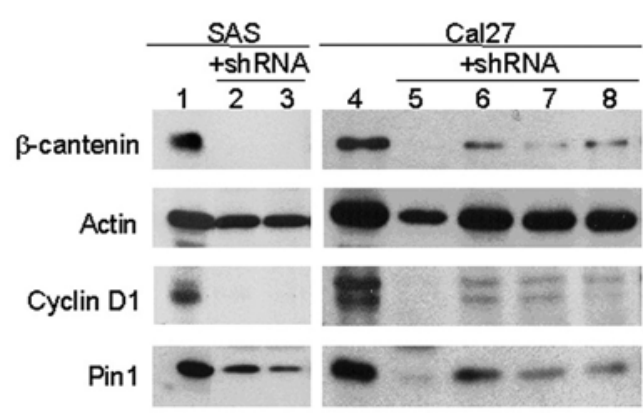

Figure 4. Expression of Pin1, ß-catenin and cyclin D1 in Pin1-transfected and Pin 1/shRNA transfected OSCC cells lines. Assessment of Pin 1 Bcatenin and cyclin D1 in four oral cancer cell lines. Increase in cellular B-catenin and cyclin D1 protein by Pin1. Four oral cancer cell lines were transfected or non-transfected with Pin1, followed by immunoblotting analysis of the cell lysates with antibodies against Pin $1 B$-catenin and cyclin D1, with actin as a control. Cells were transient transfected with 4 $\mu \mathrm{g}$ of Pin $1 / \mathrm{shRNA}$ according to the manufacturer's protocol. At 2 days posttransfection, the medium was replaced with fresh growth medium without antibiotic containing puromycin at $3 \mu \mathrm{g} / \mathrm{ml}$ for clonal selection. Selection was maintained in a medium containing puromycin at $2 \mu \mathrm{g} / \mathrm{ml}$ to ensure a stable transfection.

confirmed by Pin 1 depletion experiment by shRNA described below. The shRNA of Pin1 was used to deplete the endogenous Pin1 level in two OSCC cell lines, SAS and Cal27. As shown in Fig. 4B, the results showed that inhibition of both $\beta$-catenin accumulation and downregulation of cyclin D1 levels accompany the Pin 1 suppression in either stable clone or transient transfection experiment. Taken together, our results demonstrated overexpression of Pin 1 up-regulated $\beta$-catenin and cyclin D1 levels in a direct manner and this might play an important role in OSCC tumorigenesis.

Relationships between expression pattern of Pin 1 and pathological cell differentiation and prognosis in OSCC. We finally compared Pin 1 expression pattern with clinicopathological parameters in 73 OSCC patients as described in detail in Materials and methods. We were particularly interested in whether the Pin 1 expression level can be used as a prognostic marker for OSCC. By immunohistochemistry examination, Pin 1 protein expression levels and extent in oral cancer paraffin sections were scored according to the Pin1 staining intensity in the nucleus and the extent of staining of tumor cells (17). We defined the Pin1 overexpression based on the Pin 1 staining score ranking $3(+++)$ or above, whereas the non-Pin 1 overexpression was of the score ranking from 0 $(+/-)$ to $2(++)$. As shown in Fig. 5, the results from KaplanMeier curves analysis revealed a significant difference among

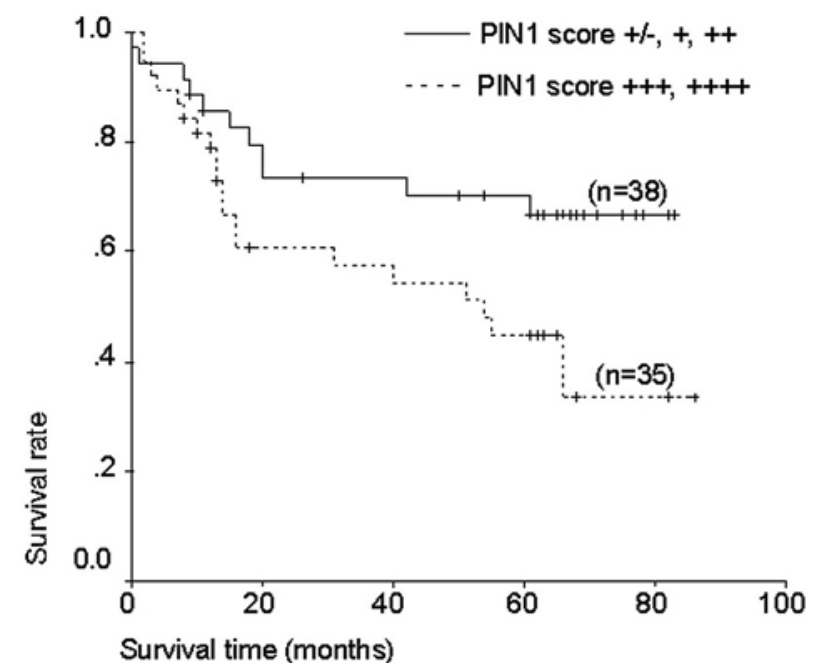

Figure 5. The relationship between disease-free survival and the Pin 1 IHC score of non-tumor part. The dot-plot line represents overexpression of Pin1. Kaplan-Meier survival curves of patients with oral squamous cell carcinoma according to expression pattern of Pin1. The survival curves were analyzed by the log-rank test. Overall survival was calculated from the time of surgery to date of death, as the event of interest, or the date of last follow-up. All statistical analysis was performed using SPSS software.

the groups overexpression and non-overexpression of Pin1. The patients who showed higher scores in the expression of Pin1 had significantly poorer survival than those who showed the lower score of Pin 1 expression $(\mathrm{P}<0.0001$ by logrank test; Fig. 5). These results strongly suggested that Pin1 expression level might be a good prognosis marker for OSCC.

\section{Discussion}

Previously, Bao et al, showed Pin 1 overexpression in 38 types of human cancers with elevated Pin1 levels relative to the corresponding normal tissue including most common human cancers such as prostate, cervical, brain, lung, breast, liver cancer, and melanoma (17). However, they did not determine the levels of Pin 1 expression in either head and neck cancers or oral cancers. In this study, we demonstrated that Pin1 was overexpressed in oral squamous cell carcinoma (OSCC) by immunoblotting and immunohistochemistry analysis (Figs. 1 and 2). These results indicated that Pin1 overexpression is prevalent and specific in a large subset of human cancers including OSCC.

Pin1 overexpression can activate multiple oncogenic pathways and contribute to cell transformation. Recent studies have demonstrated that Pin 1 increased the transcription level of several oncogenes including cyclin D1 and c-myc as well as conferred anchorage-independent cell growth to normal mammary epithelial cells $(16,34)$. Breast cancer for example, Pin 1 protein overexpression can lead to cyclin D1 elevation and transformation of breast epithelial cells $(16,34)$. Interestingly, a number of evidence has demonstrated that Pin1 might play an essential role in regulating the expression and turnover of cyclin D1 by multiple mechanisms. Pin1 cooperates with c-Jun N-terminal kinase (JNK) or Ha-Ras increasing transcriptional activity of phosphorylated c-Jun to activate the cyclin D1 promoter $(16,34)$. In addition, Pin1 
can bind to phosphorylated $\beta$-catenin that consequentially enhances the transcriptional activity towards its target gene, cyclin D1 $(2,15)$. Moreover, the interaction of Pin1 and phosphorylated p65/RelA subunit of NFkB prevents $\mathrm{NF \kappa B}$ from binding to I $\mathrm{B}$ that increases its transcriptional activity and consequentially elevate the cyclin D1 level (36). Besides upregulation of the cyclin D1 gene expression, Pin 1 has been demonstrated to directly bind to pT286-Pro motif of cyclin D1 and resulted in increasing its protein stability (37). This evidence demonstrates that Pin 1 overexpression can cause cyclin D1 protein elevation though at least four distinct mechanisms.

Our results showed that Pin 1 is overexpressed in oral squamous cell carcinoma (OSCC) and statistic analysis revealed that its level correlated with cyclin D1 expression (Figs. 1 and 3). Together with the results from a previous study that mice lacking Pin 1 has phenotypes remarkably similar to those in cyclin D1-deficient mice (37), these results indicated that Pin 1 is a regulator of cyclin D1 expression in OSCC and might play a role in OSCC. Indeed, evidence as described below has showed that cyclin D1 elevation is tightly associated with OSCC. First, cyclin D1 protein is overexpressed in up to $80 \%$ of head and neck SCC (23-25). Second, suppression of cyclin D1 has been shown to inhibit the SCC growth in vitro and in vivo that leads to cellular apoptosis (26). Third, overexpression of cyclin D1 has been found associated with a more aggressive phenotype and reduced survival in patients with head and neck SCC including tongue cancer (27-31). The above evidence together with our results in this study suggest that cyclin D1 might play an important role during tumorigenesis of OSCC.

For the pathological mechanism of how Pin 1 elevated cyclin D1 expression in OSCC, we have examined the plausibility of Pin1-ß-catenin-cyclin D1 signaling. Compelling evidence shows that Pin 1 mediated $\beta$-catenin accumulation and enhanced its transcriptional activity in both in vitro and in vivo conditions. By using an antisense approach, Ryo et al, showed that down-regulation of Pin1 in HeLa cells led to a decrease in $\beta$-catenin levels (15). In Pin1 knockout mice, lower B-catenin levels were found in all tissues compared to those of wild-type mice $(15,34)$. In addition, Pin1 has been demonstrated to bind directly to phosphorylated B-catenin at pT246P motif in vitro and in vivo and block the interaction between $\beta$-catenin and APC $(15,34)$. Moreover, Pin1- $\beta$-catenin signaling has been shown to regulate cyclin D1 expression in human breast and liver cancers when Pin1 was overexpressed (16-20). These studies suggested a mechanism that overexpression of Pin 1 contributes to the $\beta$-catenin accumulation and increases the nuclear fraction of $\beta$-catenin by preventing its interaction with APC in tumors that consequently elevates the cyclin D1 gene expression. It is noteworthy that both APC and $\beta$-catenin mutations are not common in breast and liver cancers. In head and neck SCC, $\beta$-catenin mutations or deletions are not common suggesting that other undefined mechanisms are involved in the B-catenin stabilization and accumulation (40). Our study showed that Pin1 protein upregulation was correlated with both $\beta$-catenin and cyclin D1 expression levels in clinical primary tumors and four oral cancer cell lines (Figs. 3 and 4A). Directly, we also demonstrated that overexpression of Pin 1 by transfection of Pin1 gene in OSCC cells can significantly elevate the protein levels of B-catenin and cyclin D1 in oral cancer cell lines (Fig. 4A). Furthermore, the results of cancer cells transfected with shRNA of Pin1 caused significant suppression of Pin1 expression, reduction of B-catenin accumulation as well as cyclin D1 level reduction (Fig. 4B). Taking these data together, we believed that Pin1-ß-catenin-cyclin D1 signaling played a role in OSCC tumorigenesis.

It has been reported that the pathological molecular mechanism that overexpression of Pin 1- $\beta$-catenin-cyclin D1 overexpression can be used as a marker of poor prognosis in human cancers including breast, prostate and liver $(16,19)$. Therefore, Pin 1 overexpression might also be used as an indicator for pathological diagnosis or prognosis in OSCC. We analyzed the association of Pin1 protein level with clinicopathological parameters and our results showed Pin 1 overexpression was correlated with pathological cell differentiation status in OSCC (Fig. 2). Together with Kaplan-Meier curves analysis revealed a significant difference between Pin1 overexpression and non-Pin 1 overexpression OSCC patients (Fig. 5). These results strongly suggested that Pin1 protein level might be a good diagnosis and prognosis marker for OSCC.

The prevalent overexpression of Pin 1 in human cancers and correlation of overexpression with poor clinical outcomes in oral cancer patients are consistent with the previous findings that Pin 1 overexpression plays specific roles in oncogenesis $(1,17)$. Furthermore, several lines of evidence including ours strongly suggested that inhibition of Pin 1 could suppress oncogenesis that provides a new avenue for anti-cancer therapy. Pin 1 is an enzyme with high specificity that can potentiate the function of many oncogenic pathways (1-4). Pin1 overexpression can confer transforming properties on mammary epithelial cells that is associated with tumorigenesis (34). In addition, anti-sense Pin1 or dominantnegative Pin 1 causes Pin1 depletion and apoptosis in cancer cells that indicates inhibition of Pin 1 activity might kill cancer cells $(33,34)$. Furthermore, the strong relationship between the Pin1 level and the clinical outcome of oral (Fig. 5) and prostate cancers, suggests that Pin 1 could become a potential therapeutic target in patients with biologically aggressive tumors (18). Finally, structurally and functionally, Pin1 can be divided into two domains, PPIase and WW domain, which can serve as chemical intervention targets (6-8). It is reasonable to assume that chemical intervention on either PPIase activity or the phosphoprotein binding activity of WW domain (for example: phosphorylated cyclin D1, - Bcatenin, -NFKB and -c-Jun) might block the oncogenic signaling $(1,2)$.

In conclusion, our data suggest that the level of Pin 1 expression may be clinically useful for assessing prognosis, particularly in primary oral carcinoma specimens, warranting prospective confirmation. Furthermore, our findings suggest that Pin1 might be a useful target for new drug development.

\section{Acknowledgements}

We would like to thank the Grant support from National Science Council (NSC93-2320-B075B-008 to P.J.L.), Kaohsiung Veterans General Hospital (VGHKS94-057 to 
P.J.L.), and VTY Joint research Program, Tsou's Foundation, Taipei, Taiwan. We also thank Dr Mark Kuo in the Department of Dentistry, National Taiwan University Hospital, for his generous gifts of oral cancer cell lines.

\section{References}

1. Lu KP, Liou YC and Zhou XZ: Pinning down the proline-directed phosphorylation signaling. Trends Cell Biol 12: 164-172, 2002.

2. Wulf G, Finn G, Suizu F and Lu KP: Phosphorylation-specific prolyl isomerization: is there an underlying theme? Nat Cell Biol 7: 435-441, 2005.

3. Lu KP, Hanes SD and Hunter T: A human peptidyl-prolyl isomerase essential for regulation of mitosis. Nature 380: 544-547, 1996.

4. Yaffe MB, Schutkowski M, Shen M, Zhou XZ, Stukenberg PT, Rahfeld J, Xu J, Kuang J, Kirschner MW, Fischer G, Cantley LC and Lu KP: Sequencespecific and phosphorylation-dependent proline isomerization: a potential mitotic regulatory mechanism. Science 278: 1957-1960, 1997.

5. Winkler KE, Swenson KI, Kornbluth S and Means AR: Requirement of the prolyl isomerase Pin1 for the replication checkpoint. Science 287: 1644-1647, 2000

6. Ranganathan R, Lu KP, Hunter T and Noel JP: Structural and functional analysis of the mitotic peptidyl-prolyl isomerase Pin1 suggests that substrate recognition is phosphorylation dependent. Cell 89: 875-886, 1997.

7. Lu PJ, Zhou XZ, Shen M and Lu KP: A function of WW domains as phosphoserine- or phosphothreonine-binding modules. Science 283: 1325-1328, 1999.

8. Verdecia MA, Bowman ME, Lu KP, Hunter T and Noel JP: Structureal basis for phosphoserine-proline recognition by group IV WW domain. Nat Struct Biol 7: 639-643, 2000.

9. Shen M, Stukenberg PT, Kirschner MW and Lu KP: The essential mitotic peptidyl-prolyl isomerase Pin 1 binds and regulates mitosis-specific phosphoproteins. Genes Dev 12: 706-720, 1998.

10. Zacchi P, Gostissa M, Uchida T, Salvagno C, Avolio F, Volinia S, Ronai Z, Blandino G, Schneider C and Del Sal G: The prolyl isomerase Pin 1 reveals a mechanism to control p53 functions after genotoxic insults. Nature 419: 853-857, 2002.

11. Zheng H, You H, Zhou XZ, Murray SA, Uchida T, Wulf G, Gu L, Tang X, Lu KP and Xiao ZX: The prolyl isomerase Pin 1 is a regulator of p53 in genotoxic response. Nature 419: 849-853, 2002

12. Lu PJ, Wulf G, Zhou XZ, Davies P and Lu KP: The prolyl isomerase Pin1 restores the function of Alzheimer-associated phosphorylated tau protein. Nature 419: 784-788, 1999.

13. Zhou XZ, Kops O, Werner A, Lu PJ, Shen M, Stoller G, Küllertz G, Stark M, Fischer G and Lu KP: Pin1-dependent prolyl isomerization regulates dephosphorylation of Cdc25C and tau proteins. Mol Cell 6: 873-883, 2000.

14. Stukenberg PT and Kirschner MW: Pin 1 acts catalytically to promote a conformational change in Cdc25. Mol Cell 7: 1071-1083, 2001

15. Ryo A, Nakamura N, Wulf G, Liou YC and Lu KP: Pin1 regulates turnover and subcellular localization of beta-catenin by inhibiting its interaction with APC. Nat Cell Biol 3: 793-801, 2001.

16. Wulf GM, Ryo A, Wulf GG, Lee SW, Niu T and Lu KP: Pin1 is overexpressed in breast cancer and potentiates the transcriptional activity of phosphorylated c-Jun towards the cyclin D1 gene. EMBO J 20: 3459-3472, 2001

17. Bao L, Kimzey A, Sauter G, Sowadski JM, Lu KP and Wang DG: Prevalent overexpression of prolyl isomerase Pin1 in human cancers. Am J Pathol 164: 1727-1737, 2004

18. Ayala G, Wang DG, Wulf G, Frolov A, Li R, Sowadski J, Wheeler TM, Lu KP and Bao L: The prolyl isomerase Pin1 is a novel prognostic marker in human prostate cancer. Cancer Res 63: 6244-6251, 2003.

19. Pang R, Yuen J, Yuen MF, Lai CL, Lee TK, Man K, Poon RT, Fan ST, Wong CM, Ng IO, Kwong YL and Tse E: Pin 1 overexpression and $\beta$-catenin gene mutationare distinct ongogenic events in human hepatocellular carcinoma. Oncogene 23: 4182-4186, 2004.

20. Wulf G, Garg P, Liou YC, Iglehart D and Lu KP: Modeling breast cancer in vivo and ex vivo reveals an essential role of Pin1 in tumorigenesis. EMBO J 23: 3397-3407, 2004.
21. Baldin V, Lukas J, Marcote MJ, Pagano M and Draetta G: Cyclin D1 is a nuclear protein required for cell cycle progression in G1. Genes Dev 7: 812-821, 1993.

22. Lukas J, Pagano M, Staskova Z, Draetta G and Bartek J: Cyclin D1 protein oscillates and is essential for cell cycle progression in human tumor cell lines. Oncogene 9: 707-718, 1994.

23. Xu L, Davidson BJ, Murty VV, Li RG, Sacks PG and Garin-Chesa P: TP53 gene mutations and CCND1 gene amplification in head and neck squamous cell carcinoma cell lines. Int J Cancer 59: 383-387, 1994.

24. Bartkova J, Lukas J, Muller H, Strauss M, Gusterson B and Bartek J: Abnormal patterns of D-type cyclin expression and G1 regulation in human head and neck cancer. Cancer Res 55: 949-956, 1995.

25. Adelaide J, Monges G, Derderian C, Seitz JF and Birnbaum D: Oesophageal cancer and amplification of the human cyclin $\mathrm{D}$ gene CCND1/PRAD1. Br J Cancer 71: 64-68, 1995.

26. Sauter ER, Nesbit M, Litwin S, Klein-Szanto AJ, Cheffetz S and Herlyn M: Antisense cyclin D1 induces apoptosis and tumor shrinkage in human squamous carcinomas. Cancer Res 59: 4876-4881, 1999.

27. Masuda M, Hirakawa N, Nakashima T, Kuratomi Y and Komiyama S: Cyclin D1 overexpression in primary hypopharyngeal carcinomas. Cancer 78: 390-395, 1996.

28. Akervall JA, Michalides RJ, Mineta H, Balm A, Borg A and Dictor MR: Amplification of cyclin D1 in squamous cell carcinoma of the head and neck and the prognostic value of chromosomal abnormalities and cyclin D1 overexpression. Cancer 79: 380-389, 1997

29. Kyomoto R, Kumazawa H, Toda Y, Sakaida N, Okamura A and Iwanaga M: Cyclin-D1-gene amplification is a more potent, prognostic factor than its protein overexpression in human headand-neck squamous-cell carcinoma. Int J Cancer 74: 576-581, 1997.

30. Michalides RJ, van Veelen NM, Kristel PM, Hart AA, Loftus BM and Hilgers FJ: Overexpression of cyclin D1 indicates a poor prognosis in squamous cell carcinoma of the head and neck. Arch Otolaryngol Head Neck Surg 123: 497-502, 1997.

31. Mineta H, Miura K, Takebayashi S, Ueda Y, Misawa K and Harada H: Cyclin D1 overexpression correlates with poor prognosis in patients with tongue squamous cell carcinoma. Oral Oncol 36: 194-198, 2000.

32. Nakahara Y, Shintani S, Mihara M, Kiyota A, Ueyama Y and Matsumura T: Alterations of Rb, p16 (INK4A) and cyclin D1 in the tumorigenesis of oral squamous cell carcinomas. Cancer Lett 160: 3-8, 2000.

33. Lu PJ, Zhou XZ, Liou YC, Noel JP and Lu KP: Critical role of WW domain phosphorylation in regulating phosphoserine binding activity and Pin1 function. J Biol Chem 277: 2381-2384, 2002.

34. Ryo A, Liou YC, Wulf G, Nakamura N, Lee SW and Lu KP: Pin1 is an E2F target gene essential for the Neu/Ras-induced transformation of mammary epithelial cells. Mol Cell Biol 22: 5281-5295, 2002.

35. Alt JR, Cleveland JL, Hannink M and Diehl JA: Phosphorylation-dependent regulation of cyclin D1 nuclear export and cyclin D1-dependent cellular transformation. Genes Dev 14: 3102-3114, 2000.

36. Ryo A, Suizu F, Yoshida Y, Perrem K, Liou YC, Wulf G, Rottapel R, Yamaoka S and Lu KP: Regulation of NF-kappaB signaling by Pin1-dependent prolyl isomerization and ubiquitinmediated proteolysis of p65/RelA. Mol Cell 6: 1413-1426, 2003.

37. Liou YC, Ryo A, Huang HK, Lu PJ, Bronson R, Fujimori F, Uchida T, Hunter T and Lu KP: Loss of Pin 1 function in the mouse causes phenotypes resembling cyclin D1-null phenotypes. Proc Natl Acad Sci USA 99: 1335-1340, 2002

38. Fujimori F, Takahashi K, Uchida $C$ and Uchida T: Mice lacking Pin1 develop normally, but are defective in entering cell cycle from $\mathrm{G}(0)$ arrest. Biochem Biophys Res Commun 265: 658-663, 1999.

39. Ashita MH, Uchida T, Mori S, Echigo S and Motegi K: Expression status of Pin 1 and cyclins in oral squamous cell carcinoma: Pin 1 correlates with Cyclin D1 mRNA expression and clinical significance of cyclins. Oncol Rep 10: 1045-1048, 2003.

40. Miyashita H, Mori S, Motegi K, Fukumoto M and Uchida T: Pin1 is overexpressed in oral squamous cell carcinoma and its levels correlate with cyclin D1 overexpression. Oncol Rep 10: $455-461,2003$. 